\title{
Intracerebral Monoamine Concentration after Ventriculoperitoneal Shunting in the Congenital Hydrocephalus Rat
}

\author{
Yutaka OTSUBO, Hiroshi ITO, and Takeshi SHIBUYA* \\ Departments of Neurosurgery and ${ }^{*}$ Pharmacology, Tokyo Medical College, Tokyo
}

\begin{abstract}
This study investigated the relationship between neurotransmitters and improvement of symptoms after ventriculoperitoneal shunting in congenital hydrocephalus (LEW-HYR) rats. Twenty-four patent hydrocephalus rats, aged 12-14 days, were randomly assigned to the following four groups; ventriculoperitoneal shunt group, obstructed shunt group, burr hole group, and no treatment group. In addition, six normal rats served as control group. Head to body length ratio was measured before and 7 days after the procedures. Coordination movement was evaluated on the 7 th postoperative day. High performance liquid chromatography (HPLC) was used to measure the concentrations of dopamine (DA), norepinephrine (NE), serotonin, homovanillic acid (HVA), 3-methoxyl-4-hydroxyphenylenglycol, 5hydroxy-indolacetic acid (5-HIAA), and 3,4-dihydroxyphenylacetic acid in the whole cerebral cortex, the thalamus and hypothalamus, the midbrain, the lower brainstem, the cerebellum, and the striatum. Fluorohistochemical studies were also performed. Significant improvements were observed in body proportion and coordination movement in the ventriculoperitoneal shunt group compared to the burr hole group and the no treatment group. HPLC and fluorohistochemical studies revealed that concentrations of $\mathrm{NE}$ in the thalamus and hypothalamus and DA in the striatum were significantly lower in the burr hole group and the no treatment group. Concentrations of HVA and 5-HIAA in the cerebellum were significantly lower in the control group. The present study indicates that ventriculoperitoneal shunting may improve the changes in concentrations of neurotransmitter in specific neurons caused by hydrocephalus, and this may contribute to the improvement of the symptoms.
\end{abstract}

Key words: monoamine, congenital hydrocephalus rat, ventriculoperitoneal shunt

\section{Introduction}

Ventriculoperitoneal shunting has been studied in experimental hydrocephalus models for many years. ${ }^{2,4,6,11,16,17)}$ However, these studies have mainly investigated the morphological changes after ventriculoperitoneal shunt, and have not assessed the changes in the levels of intracerebral monoamines.

The LEW-HYR congenital hydrocephalus rat was established by repeated inbreeding of Lewis rats with hydrocephalus caused by mutation. ${ }^{9)}$ LEWHYR rats have communicating hydrocephalus due to developmental abnormality of the subarachnoid space, ${ }^{10]}$ which is inherited through recessive genes. ${ }^{9)}$ If the hydrocephalus is left untreated, the rats die within 3 to 4 weeks of birth because of insufficient feed intake. ${ }^{\text {g) }}$

The present study used LEW-HYR rats as a model

Received December 9, 1996; Accepted June 2, 1997 for treatment of hydrocephalus by ventriculoperitoneal shunting and investigated the relationship between the levels of intracerebral monoamines and their metabolites and improvement of symptoms after ventriculoperitoneal shunting.

\section{Materials and Methods}

Male rats with manifest hydrocephalus, aged 12 to 14 days, were randomly divided into four groups: the untreated hydrocephalus group, the burr hole group, the obstructed ventriculoperitoneal shunt group, and the ventriculoperitoneal shunt group. Normal rats were used as the control group. Each five groups consisted of six rats.

The head to body length ratio was measured aged 12 to 14 days, and then the animals underwent various procedures. Ventriculoperitoneal shunting was performed under halothane inhalation anesthesia (Fig. 1). ${ }^{14)}$ A silicone tube with an inner diameter of 



Fig. 1 Ventriculoperitoneal shunt procedure in LEW-HYR rats, A: Under anesthesia using a mixture of halothane, oxide, and nitrous oxide, a burr hole is made in the left parietal bone. B: The peritoneum is opened. C: Ventricular tapping is performed, and the flow of cerebrospinal fluid by detention of pulsatile flow is confirmed. D: The shunt tube is then inserted into the peritoneum. E: The skin is closed. F: Body temperature maintained during the recovery from anesthesia.

0.025 inches was used for the shunt system. The obstructed ventriculoperitoneal shunt had been ligated in advance, so had the effect of tapping the ventricle, but without continuous drainage. The burr hole was also performed under general anesthesia. Seven days later, head to body length ratio was measured again, and coordination movement was evaluated by counting with the KN-75 Rota Rod (Natsume Seisakusho Co. Ltd., Tokyo). The rat brains were then extracted immediately, and each brain was divided into six parts ${ }^{5}$ : the whole cerebral cortex, the thalamus and hypothalamus, the midbrain, the lower brainstem, the cerebellum, and the striatum.

High performance liquid chromatography (HPLC) was used to measure the concentrations of norepinephrine (NE), dopamine (DA), serotonin (5-HT), and their metabolites; homovanillic acid (HVA), 3-methoxyl-4-hydroxyphenylenglycol, and 5- hydroxy-indolacetic acid (5-HIAA), and 3,4-dihydroxyphenylacetic acid. ${ }^{7}$ The non-parametric Mann-Whitney test was used for statistical analysis. Fluorohistochemical studies according to the FalckHillarp method ${ }^{3)}$ were performed for each group.

\section{Results}

Changes of body proportion as shown by the head to body length ratio before and 7 days after the procedures are shown in Fig. 2. The ratio significantly decreased in the ventriculoperitoneal shunt group from $15.73 \pm 0.28$ to $13.69 \pm 0.35$ (mean $\pm \mathrm{SE}, \mathrm{p}$ $<0.05$ ), but increased in the untreated hydrocephalus group.

Movement coordination was significantly better ( $p<0.05$ ) in the ventriculoperitoneal shunt group $[64.75 \pm 8.27]$ compared with the hydrocephalus 


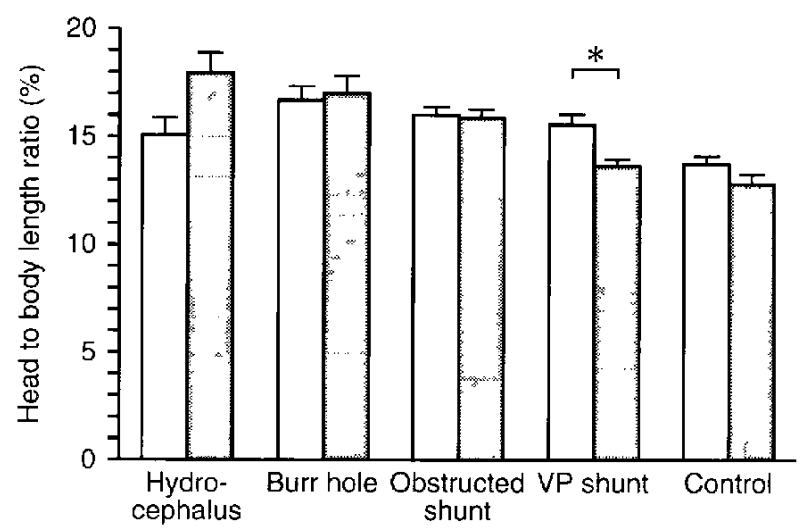

Fig. 2 Changes in body proportion shown by head to body length ratio before (open column) and 7 days after (shaded column) various procedures. Values are mean $\pm \mathrm{SE} .{ }^{*} \mathrm{p}<$ 0.05. VP: ventriculoperitoneal.

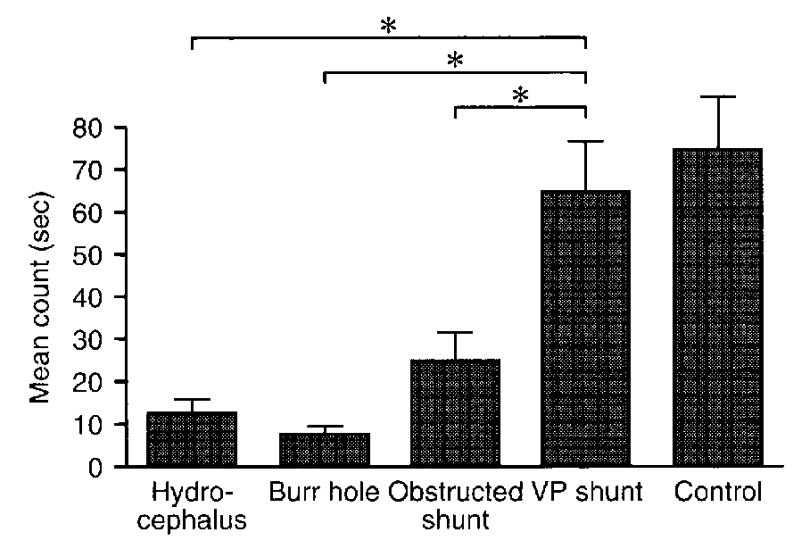

Fig. 3 Movement coordination on the 7th postoperative day. Values are mean $\pm \mathrm{SE}$. ${ }^{*} \mathbf{p}<$ 0.05. VP: ventriculoperitoneal.

group $(12.83 \pm 3.54)$, the burr hole group $(8.17 \pm$ 1.08), and the obstructed ventriculoperitoneal shunt group (25.08 \pm 6.55$)$ (mean \pm SE) (Fig. 3). There were no significant differences between the other groups, although movement coordination was slightly better in the obstructed ventriculoperitoneal shunt group.

HPLC measurement of the concentrations of monoamines and their metabolites showed that the concentrations of 5-HIAA in the whole cerebral cortex were significantly lower in the hydrocephalus group (178.62 $\pm 6.71 \mathrm{ng} / \mathrm{g})$ and the burr hole group $(150.88 \pm 2.45 \mathrm{ng} / \mathrm{g})$ (mean $\pm \mathrm{SE}, \mathrm{p}<0.05)$ compared with the ventriculoperitoneal shunt group (Fig. 4 upper). No significant difference was found between the groups in the midbrain (Fig. 4 middle).
The concentrations of HVA were significantly lower in the control group (59.44 $\pm 13.76 \mathrm{ng} / \mathrm{g}$ ) (mean \pm $\mathrm{SE}, \mathrm{p}<0.05)$ compared with the hydrocephalus group in the cerebellum. The concentrations of 5HIAA were significantly lower in the ventriculoperitoneal shunt group (196.52 $\pm 16.02 \mathrm{ng} / \mathrm{g})$ and the control group (176.54 $\pm 14.44 \mathrm{ng} / \mathrm{g}$ ) (mean $\pm \mathrm{SE}, \mathrm{p}<0.05$ ) compared with the hydrocephalus group (Fig. 4 lower).

The concentrations of NE in the thalamus and hypothalamus were significantly lower in the hydrocephalus group (1286.44 $\pm 168.14 \mathrm{ng} / \mathrm{ml})$ and burr hole group $(1188.91 \pm 130.20 \mathrm{ng} / \mathrm{g}$ ) (mean \pm SE, $p<0.05)$ compared with the ventriculoperitoneal shunt group (Fig. 5 upper). There were no significant differences between the groups in the lower brainstem including the locus ceruleus and the raphe nucleus (Fig. 5 middle). The concentrations of DA in the striatum were significantly lower in the hydrocephalus group $(1460.00 \pm 38.98 \mathrm{ng} / \mathrm{g})$ and the burr hole group (1074.44 $\pm 152.08 \mathrm{ng} / \mathrm{g}$ ) (mean $\pm \mathrm{SE}, \quad \mathrm{p}<0.05$ ) compared with the ventriculoperitoneal shunt group (Fig. 5 lower).

The fluorohistochemical study showed that the fluorescence of the NE neurons in the median eminence was less in the hydrocephalus group compared with the ventriculoperitoneal shunt group (Fig. 6 upper row). There was no clear difference in fluorescence in the locus ceruleus between the hydrocephalus group and ventriculoperitoneal shunt group (Fig. 6 middle row). Fluorescence in the striatum was less at the terminal portion of the DA neurons in the hydrocephalus group (Fig. 6 lower row). Fluorescence of the monoamine neurons was also less adjacent to the ventricular system.

\section{Discussion}

Few studies of ventriculoperitoneal shunt in experimental hydrocephalus have demonstrated the patency or the adequacy of the shunts. ${ }^{2,4,6,11,16,17]}$ The present study showed that body proportion and movement coodination improved after shunt procedure, suggesting that this model is suitable for assessing hydrocephalus treatment. The cause of hydrocephalus in LEW-HYR is developmental abnormality of the subarachnoid space. ${ }^{9,10)}$ However, the results also show that hydrocephalus can be treated if the ventriculoperitoneal shunting is performed at an early stage after birth.

The concentrations of intracerebral monoamines and their metabolites under the hydrocephalic conditions have been investigated..$^{1,8,12,13,15)}$ Chovanes et al. ${ }^{1)}$ reported that the concentrations of NE, DA, and 5-HT are significantly lower in the cortex, the 

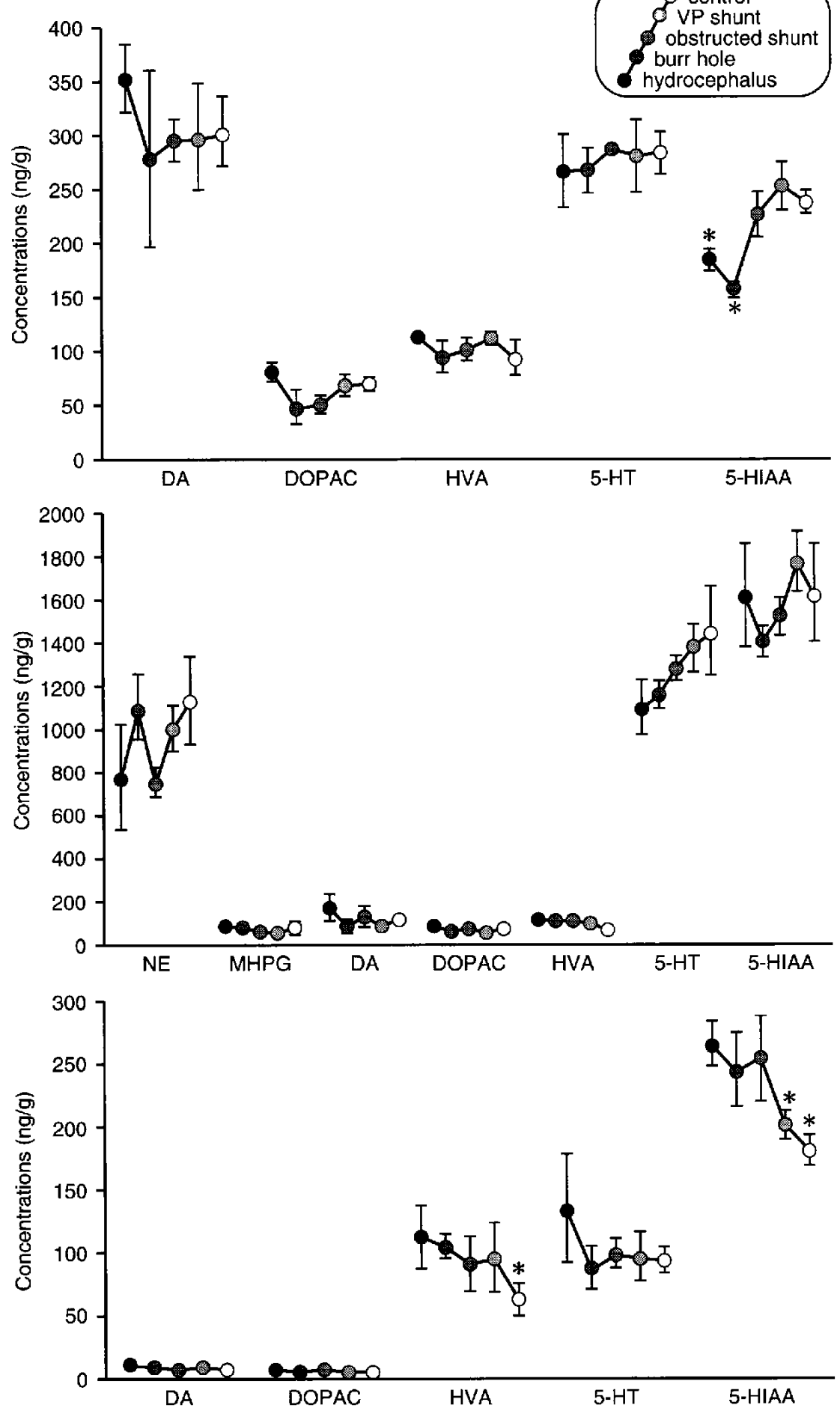

Fig. 4 Concentrations of intracerebral monoamines and their metabolites in each group measured by high performance liquid chromatography. upper: In the whole cerebral cortex, concentrations of 5-hydroxy-indolacetic acid (5-HIA A) were significantly lower in the hydrocephalus group and burr hole group compared with the ventriculoperitoneal (VP) shunt group $\left({ }^{*} p<0.05\right)$. middle: In the midbrain, no significant difference was noted between the groups. lower: In the cerebellum, the concentrations of homovanillic acid (HVA) were significantly lower in the control group compared with the hydrocephalus group, and concentrations of 5 HIAA were significantly lower in the VP shunt group and the control group compared with the hydrocephalus group $\left({ }^{*} p<0.05\right)$. Values are mean \pm SE. NE: norepinephrine, DA: dopamine, DOPAC: 3,4-dihydroxyphenylacetic acid, 5-HT: serotonin, MHPG: 3-methoxyl-4-hydroxyphenylenglycol. 

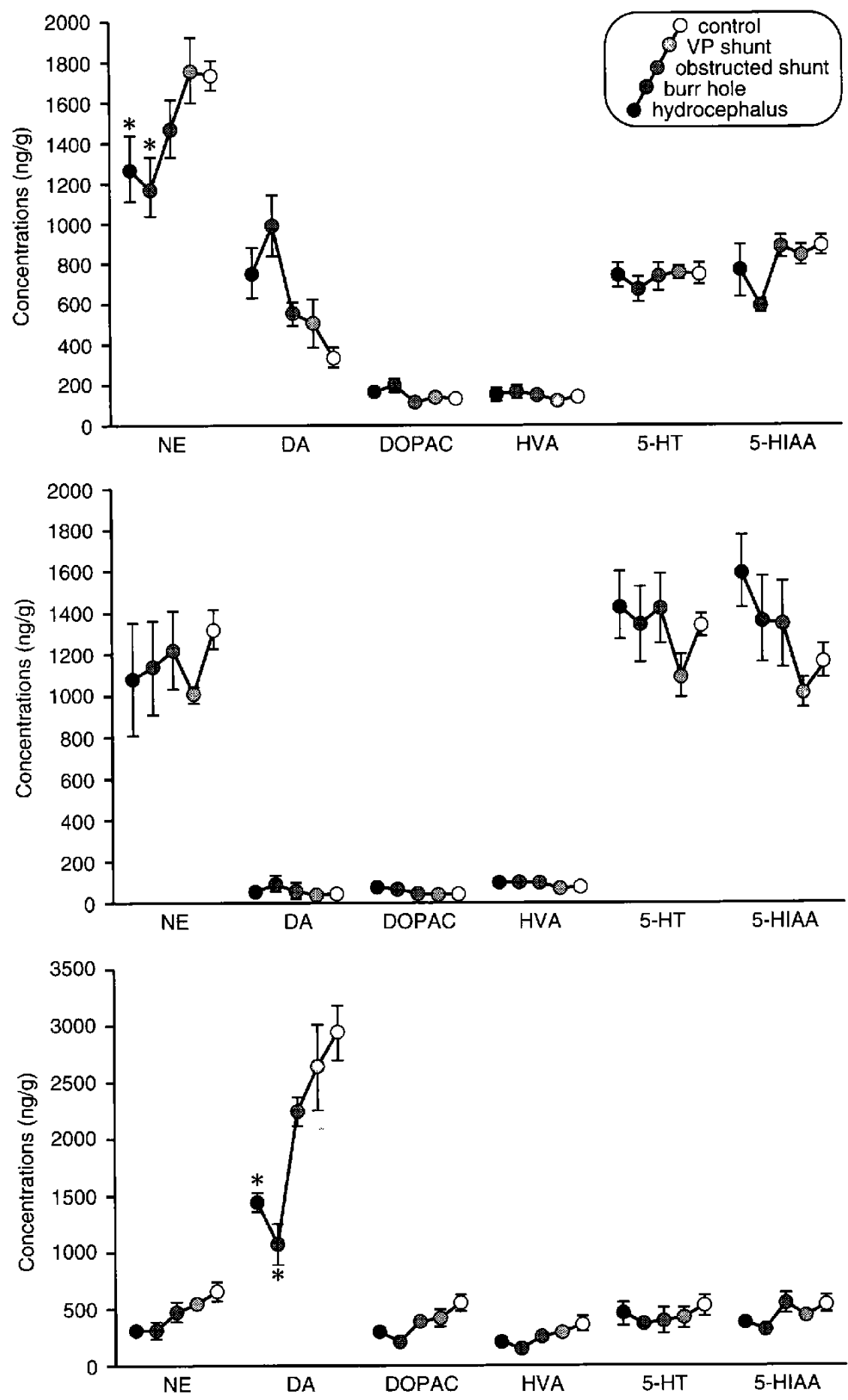

Fig. 5 Concentrations of monoamines and their metabolites measured by high performance liquid chromatography. upper: In the thalamus and hypothalamus, concentrations of norepinephrine (NE) were significantly lower in the hydrocephalus group and burr hole group compared with the ventriculoperitoneal (VP) shunt group $\left({ }^{*} p<0.05\right)$. middle: In the lower brainstem, there was no significant difference between the groups. lower: In the striatum, concentrations of dopamine (DA) were significantly lower in the hydrocephalus group and the burr hole group compared with the VP shunt group $\left({ }^{*} p<0.05\right)$. Values are mean \pm SE. DOPAC: 3,4-dihydroxyphenylacetic acid, HVA: homovanillic acid, 5-HT: serotonin, 5HIAA: 5-hydroxy-indolacetic acid. 


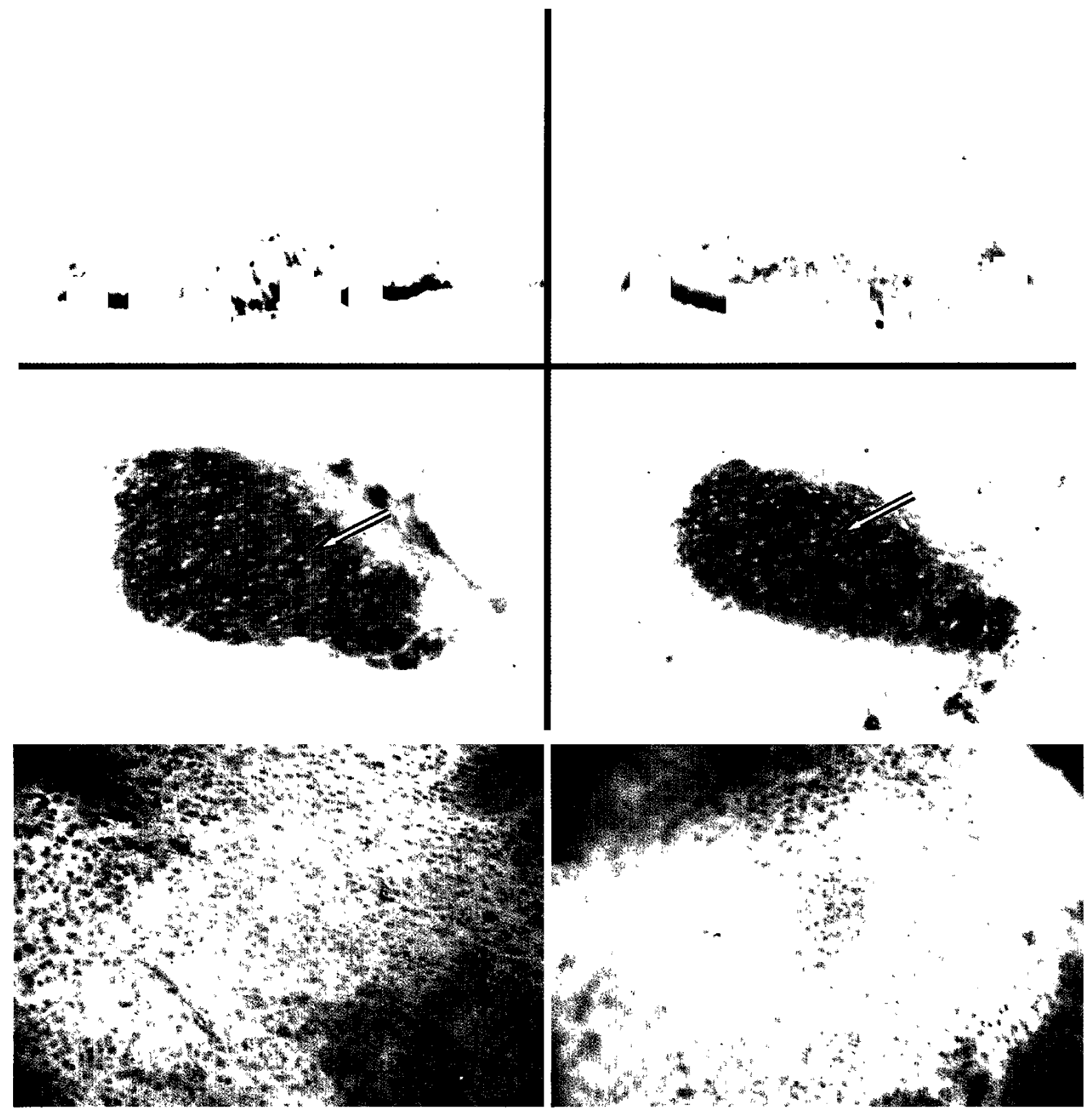

Fig. 6 Fluorohistochemical study according to the Falck-Hillarp method. upper row: In the median eminence, fluorescence of the norepinephrine neurons was less in the hydrocephalus group (left) compared with the ventriculoperitoneal shunt group (right). middle row: In the locus ceruleus (arrow), there was no clear difference in fluorescence between the hydrocephalus group (left) and the ventriculoperitoneal shunt group (right). lower row: In the striatum, fluorescence was less at the terminal portion of the dopamine neurons in the hydrocephalus group (left) and the ventriculoperitoneal shunt group (right).

striatum, and the cerebellum in neonatal rats with kaolin-induced hydrocephalus, and Miyake et al. ${ }^{13 j}$ pointed out that the concentrations of NE and DA are significantly lower in the whole brain in rats with kaolin-induced hydrocephalus.

The present HPLC and fluorohistochemical stud- ies showed that the levels of DA in the striatum and $\mathrm{NE}$ in the thalamus and hypothalamus were significantly lower in the congenital hydrocephalus (LEW-HYR) rat. The concentrations of monoamine decreased in specific neurons, but no significant change was noted in the metabolites. Specifically, 
production of monoamines decreased in specific neurons in the LEW-HYR rat, but there was no extensive effect on the turnover of monoamines. These disturbances were dramatically improved by ventriculoperitoneal shunting. This suggests that the changes of DA neurons in the striatum and changes of NE neurons in the thalamus and hypothalamus are not hypoplasia in the embryological sense, but secondary dysfunction of neurons caused by hydrocephalus. Some of the diverse clinical symptoms caused by hydrocephalus are considered to be caused by secondary dysfunction in these specific neurons. The present study found decreased monoamine levels in tissues near the ventricular system. This suggests that these changes were caused by increased intraventricular pressure and the inflow of cerebrospinal fluid into the brain tissue. The concentrations of NE in the locus ceruleus and 5-HT in the raphe nucleus did not change significantly after ventriculoperitoneal shunting. The present model suggests that the influence of hydrocephalus on the brainstem is low, occurring only in the terminal stage as in clinical hydrocephalus.

The concentrations of some monoamines were higher in the hydrocephalus group than in the shunt group. In the cerebellum, the concentrations of HVA and 5-HIAA were significantly higher in the hydrocephalus group compared with the control group. This may be attributed to the changes in monoamine turnover due to hydrocephalus, or to the influence of cerebrospinal fluid in the brain tissue. In the thalamus and hypothalamus, DA exhibited higher values in the hydrocephalus group and burr hole group compared with the control group (Fig. 5 upper). The differences were not statistically significant, but a trend contrary to that of DA neurons in the striatum was noted. Neurotransmitters may accumulate in specific neurons in LEW-HYR rat without being metabolized. This may be considered as a disorder of monoamine turnover, but there are still some unclear points such as the absence of change in metabolite concentrations. The reason for the contrary trend concentrations in DA neurons at different sites will continue to be studied.

Clinically, improvement of symptoms and reduction in the size of the ventricle are observed after ventriculoperitoneal shunting. The morphological change of brain tissues and the ventricular system, which simultaneously occur with the changes in the level of intracerebral monoamines, are a topic for further study.

This model for ventriculoperitoneal shunting in congenital hydrocephalus (LEW-HYR) rats showed that changes of monoamine concentration were observed in specific neurons, and appeared to be sec- ondary changes induced by hydrocephalus. The concentrations of monoamines showed dramatic improvement after ventriculoperitoneal shunting, suggesting that this procedure may improve the changes in neurotransmitter concentration in specific neurons, thus contribute to the improvement of symptoms.

\section{References}

1) Chovanes GI, McAllister JP, Lamperti AA, Salotto AG, Truex RC Jr: Monoamine alterations during experimental hydrocephalus in neonatal rats. Neurosurgery 22: 86-91, 1988

2) Del Bigio MR, Bruni JE: Changes in periventricular vasculature of rabbit brain following induction of hydrocephalus and after shunting. I Neurosurg 69: 115-120, 1988

3) Falck B, Hillarp NA, Thieme G, Torp A: Fluorescence of catecholamines and related compounds condensed with formaldehyde. $J$ Histochem Cytochem 10: 348-360, 1962

4) Fried A, Shapiro K, Takei F, Cohn I: A labolatory model of shunt-dependent hydrocephalus. Development and biomechanical characterization. $J$ Neurosurg 66: 734-740, 1987

5) Glowinski J, Iversen LL: Regional studies of catecholamines in the rat brain-I. J Neurochem 13: 655-669, 1966

6) Granholm L: Induced reversibility of ventricular dilatation in experimental hydrocephalus. Acta Neurol Scand 42: 581-588, 1966

7) Hefti F: A simple, sensitive method for measuring 3,4-dihydroxyphenylacetic acid and homovanillic acid in rat brain tissue using high-performance liquid chromatography with electrochemical detection. Life Sci 25: 775-782, 1979

8) Higashi K, Asahisa H, Ueda N, Kobayashi K, Hara K, Noda Y: Cerebral blood flow and metabolism in experimental hydrocephalus. Neurol Res 8: 169-176, 1986

9) Higashi K, Asahisa $H$, Ueda N, Noda $Y$, Tashiro M: [An experimental model of congenital hydrocephalus in the rat]. Shoni No Noshinkei 9: 257-264, 1984 (Jpn, with Eng abstract)

10) Higashi $K$, Noda $Y$, Mifune $H$ : [Pathological studies on the brain of congenital hydrocephalic rats]. Shoni No Noshinkei 12: 1-9, 1987 (Jpn, with Eng abstract)

11) Hochwald GM, Epstein F, Malhan C, Ransohoff J: The relationship of compensated to decompensated hydrocephalus in the cat. $J$ Neurosurg 39: 694-697, 1973

12) Miwa S, Inagaki $C$, Fujiwara M, Takaori S: The activities of noradrenergic and dopaminergic neuron systems in experimental hydrocephalus. J Neurosurg 57: 67-73, 1982

13) Miyake H, Eghwrudjakpor PO, Sakamoto T, Mori K: Catecholamine alterations in experimental hydrocephalus. Childs Nerv Syst 8: 243-246, 1992 
14) Miyaoka M, Ito $M$, Wada M, Sato K, Ishii S: Measurement of local cerebral glucose utilization before and after V-P shunt in congenital hydrocephalus in rats. Metab Brain Dis 3: 125-132, 1988

15) Owman $G$, Rosengren $\mathrm{E}$, West $\mathrm{KA}$ : Influence of various intracranial pressure levels on the concentration of certain arylethylamines in rabbit brain. Experientia 27: 1036-1037, 1971

16) Rubin RC, Hochwald GM, Tiell M, Boleslaw $H L$ : Hydrocephalus-I. Histological and ultrastructual changes in the pre-shunted cortical mantle. Surg Neurol 5: 109-114, 1976

17) Rubin RC, Hochwald GM, Tiell M, Boleslaw $\mathrm{HL}$ : Hydrocephalus-II. Cell number and size, and myelin content of the pre-shunted cerebral cortical mantle. Surg Neurol 5: 115-118, 1976

Address reprint requests to: Y. Otsubo, M.D., Department of Neurosurgery, Tokyo Medical College, 6-7-1 Nishishinjuku, Shinjuku-ku, Tokyo 160, Japan.

\section{Commentary}

In the field of experimental hydrocephalus, morphological studies and cerebrospinal fluid dynamic studies have been the main focus until now. In spite of those energetic studies on hydrocephalus, we have many unresolved questions about hydrocephalic insults and recovery after shunting procedure.

This study has two main subjects: the establishment of a communicating hydrocephalic rat model and the investigation of monoamine concentration changes measured pre- and post-shunting procedure by the latest method.

In recent years, it has been disclosed that alteration of neurotransmitters plays an important role in the manifestation of symptoms due to the hydrocephalic state. Regarding monoamines, some reports showed low concentrations of NE, DA, and 5-HT in kaolin-induced hydrocephalic rats. In this study, more precise data was examined, and showed concentration differences by location. Though the authors demonstrated clearly these changes of monoamine concentration, further investigation will be necessary for data elucidation.

The newly developed model of hydrocephalus and the methodology of monoamine investigation will contribute greatly to investigations in this field.

Koki KADOTA, M.D. Department of Neurosurgery Faculty of Medicine Kagoshima University Kagoshima, Japan
Dr. Otsubo, Professors Ito and Shibuya should be complimented on their novel work described in this extensive experimental research. The nature of hydrocephalic insults on the brain metabolism has been well demonstrated by Professor Ito and his coworkers in the past. This experimental investigation delineated the nature of functional development in the congenital hydrocephalus animal model (LEWHYR rat) with the significance of CSF shunting in the infantile period, which is compatible with the stages of dendritic maturation or axonal maturation with/ without myelination. The authors performed ventriculoperitoneal shunting on hydrocephalic rats at 12 to 14 days of age with their splendid experimental technique. Hydrocephalic state is likely far-advanced in this period associated with not only significant morphological changes but also psychomotor developmental delay. The intracerebral level of monoamines and their metabolites are an excellent indicator for the neuronal maturation, especially in these periods of dendritic and axonal maturation. Although the authors found that CSF shunting improves the changes in concentration of neurotransmitter in some specific neurons caused by hydrocephalus, as shown in both quantitative measurement by high performance liquid chromatography and morphological analysis in fluorohistochemical study, the efficacy is limited in certain groups of neurotransmitters with some regional differences. These results are extremely important to standardize the reversibility of psychomotor development in the individual and the time specificity of the treatment in congenital communicating hydrocephalus, when treated with postnatal shunt. These clinicallycomparable problems have been investigated in various types of congenital hydrocephalus models, such as LEW/Jms as the model of aqueductal stenosis and HTX as the communicating hydrocephalus with/ without secondary aqueductal stenosis. ${ }^{1)}$ I am convinced that Professor Ito and his co-workers will advance their research with their highly recognized experimental knowledge and technique to overcome these clinical limitations of treatment for the congenital/intractable hydrocephalus in the future.

\section{Reference}

1) Oi S, Yamada H, Sato O, Matsumoto S: Experimental models of congenital hydrocephalus and comparable clinical problems in the fetal and neonatal periods. Childs Nerv Syst 12: 292-302, 1996

Shizuo OI, M.D. Department of Neurosurgery Tokai University School of Medicine Kanagawa, Japan 